\title{
Clinical Characteristics and Outcomes of Non-small Cell Lung Cancer Patients with HER2 Alterations in Korea
}

\author{
Kangkook Lee, $\mathrm{MD}^{1}$ \\ Hyun Ae Jung, MD2 \\ Jong-Mu Sun, MD, PhD² \\ Se-Hoon Lee, MD, $\mathrm{PhD}^{2}$ \\ Jin Seok Ahn, MD, PhD² \\ Keunchil Park, MD, PhD² \\ Myung-Ju Ahn, MD, PhD²
}

${ }^{1}$ Division of Hematology-Oncology, Department of Medicine, Dongsan Medical Center, Keimyung University School of Medicine, Daegu, ${ }^{2}$ Division of Hematology-Oncology, Department of Medicine, Samsung Medical Center, Sungkyunkwan University School of Medicine, Seoul, Korea

\begin{abstract}
Purpose
Human epidermal growth factor receptor 2 (HER2) alterations are found in approximately $1 \%-3 \%$ of non-small cell lung cancers (NSCLCs). We evaluated the clinical features and outcomes of NSCLC harboring HER2 alteration detected by next-generation sequencing (NGS) in Korea.
\end{abstract}

\section{Materials and Methods}

A total of 1,108 patients who were diagnosed with NSCLC between December 2015 and December 2017 were screened and analyzed by NGS. Medical records were reviewed retrospectively to analyze the clinical characteristics and outcomes from various treatments.

Results

HER2 alterations were identified in 36 NSCLC patients. Of the patients, 22 (61.1\%) had an exon 20 in-frame insertion mutation, 15 (41.7\%) had HER2 amplification, and one had both. The median patient age was 58 years, $55.6 \%$ were male, and $50.0 \%$ were neversmokers. Adenocarcinoma was predominant (88.9\%). The most common metastatic site was bone $(58.3 \%)$, and $66.7 \%$ of patients were stage IV at initial diagnosis. Six patients (16.7\%) had a coexistent sensitizing epidermal growth factor receptor (EGFR) mutation, and two patients (5.6\%) had anaplastic lymphoma kinase (ALK) rearrangement. With a median 14 months of follow-up, the median progression-free survival of first-line treatment was 6 months (95\% confidence interval, 4.172 to 7.828$)$, and median overall survival was not reached. The proportions of adenocarcinoma, never-smokers, and metastasis to the liver were higher in the exon 20 in-frame insertion mutation group, whereas coexistence of EGFR mutation was more frequently found in the HER2 amplification group.

\section{Conclusion}

HER2-altered NSCLC showed distinct clinical features. Moreover, different characteristics were identified between the HER2 in-frame insertion mutation group and the HER2 amplification group.

\author{
Correspondence: Myung-Ju Ahn, MD, PhD \\ of Medicine, Samsung Medical Center, \\ Sungkyunkwan University School of Medicine, \\ 81 Irwon-ro, Gangnam-gu, Seoul 06351, Korea \\ Tel: 82-2-3410-3452 \\ Fax: 82-2-3410-1754 \\ E-mail: silk.ahn@samsung.com \\ Received April 8, 2019 \\ Accepted July 25, 2019 \\ Published Online July 26, 2019
}

Key words

Non-small cell lung cancer, HER2 mutation, HER2 amplification

\section{Introduction}

The identification of oncogenic driver mutations has triggered the development of new treatments for non-small cell lung cancer (NSCLC). Targeted therapies have shown significant improvement of clinical outcomes in NSCLC patients with epidermal growth factor receptor (EGFR) mutation, anaplastic lymphoma kinase $(A L K)$, or c-ros oncogene1 (ROS1) gene rearrangement.

With the advent of precision medicine using next-generation sequencing (NGS), other various genetic alterations can be detected. Human epidermal growth factor receptor 2 (HER2), also known as NEU, EGFR2, or ERBB2, is a member of the EGFR family of receptor tyrosine kinases. HER2 alteration has been demonstrated to play important roles in the pathogenesis of certain human cancers, including breast cancer and gastric cancer, by activating downstream signaling 
via the phosphatidylinositide 3-kinase (PI3K)-protein kinase $\mathrm{B}(\mathrm{AKT})$ and mitogen-activated protein kinase kinase (MEK) extracellular signal-regulated kinase pathways.

HER2 alterations have emerged as novel oncogenic drivers and therapeutic targets in lung cancers [1]. The incidence of HER2 mutation in lung adenocarcinoma is $1 \%-5 \%$, and frequency of HER2 amplification is 2\%-5\% [2]. Previous studies have identified distinct clinical features of NSCLCs with HER2 alteration. Mazieres et al. [3] reported that HER2altered NSCLC shows a high proportion of adenocarcinoma $(100 \%)$, female patients $(69.2 \%)$, and never-smokers $(52.3 \%)$. Li et al. [4] also showed that HER2 alteration occurs predominantly in never-smokers $(100 \%)$ and female patients $(87.5 \%)$. In contrast, Arcila et al. [5] reported that there were no associations between HER2-altered NSCLC and sex, race, or stage at diagnosis. Given the small number of patients analyzed in previous studies, the clinical features and outcomes have not been fully evaluated in advanced NSCLC harboring HER2 alterations [6].

The aims of this study are to evaluate the characteristics and clinical outcomes of NSCLC patients harboring HER2 alteration detected by NGS.

\section{Materials and Methods}

\section{Patients}

A total of 1,108 patients who were initially diagnosed with metastatic NSCLC was screened between December 2015 and December 2017 at Samsung Medical Center. All tissue samples were analyzed by NGS. For patients with HER2 alterations, patient characteristics, histology, initial stage at diagnosis, sites of metastasis, and response to first-line systemic treatment for metastatic NSCLC were retrospectively analyzed.

\section{Identification of $H E R 2$ alteration}

HER2 gene alterations were identified by NGS. The Oncomine Focus Assay panel (Thermo Fisher Scientific, San Francisco, CA) or the CancerSCAN panel (LabGenomics, Seongnam, Korea) was used for analyzing HER2 alterations, including HER2 amplification and exon 20 in-frame insertion mutation.

CancerSCAN is a targeted sequencing platform, designed at Samsung Medical Center. This customized platform included target genes curated from the literature of requested by the researchers and clinicians and the selected genes covered variants associated with the targeted cancer therapies appro- ved by the Korean Ministry of Food and Drug Safety and U.S. Food and Drug Administration. In this study, CancerSCAN version 2 was applied, encompassing 381 cancer-related genes. The genes contained in the panel are listed in S1 Table. The panel has been validated with data demonstrating 95\% sensitivity and positive predictive value [7].

We also used Oncomine Focus Assay (OFA) panel, which simultaneously detects hundreds of variants across 52 genes relevant to solid tumors [8]. The biomarkers included were selected based on information in the Ion Torrent Oncomine Knowledgebase, one of the world's largest collections of curated oncology data. OFA has shown a precise, reproducible, sensitive, and accurate NGS assay for the detection of somatic genetic variants with nearly $99 \%$ of accuracy and specificity, which is commercially available [8].

Copy number variations (CNVs) of genes were detected using an in-house copy number caller. To identify somatic $\mathrm{CNVs}$, we calculated the mean read depth at each exon, normalized by the coverage of the target regions in that sample. This normalized read depth was further standardized by dividing by the expected coverage for a normal individual (the expected coverage at each exon was taken to be the median of the read depth at that exon across a set of normal individuals). These steps account for the variability in capture efficiency and GC content at different exons. To infer the correct copy number, the amplitude of the copy numbers were then adjusted based on the estimated purity. Copy numbers greater than four were categorized as amplifications.

\section{Statistical analysis}

Medical records were reviewed to obtain clinical information. Descriptive statistics were used to analyze the demographics, histology, incidence of various sites of metastasis, EGFR mutation, and ALK rearrangement. Statistical significance was set at $\mathrm{p}<0.05$. All analyses were conducted using SPSS ver. 24.0 (IBM Corp., Armonk, NY). Either the chi-squared test or Fisher exact test was used, as appropriate, to analyze statistical significance among patient characteristics. Progression-free survival (PFS) was calculated from the time of first systemic treatment to radiographic progression or death. Overall survival (OS) was also calculated from the time of first systemic treatment to the date of death. PFS and OS were analyzed using the Kaplan-Meier estimation method.

\section{Ethical statement}

Institutional Review Board (IRB) approval was obtained from Samsung Medical Center (SMC, Seoul, Korea, SMC 2019-03-107-001). The IRB approved waiver of informed consent. 
Table 1. Characteristics of the patients harboring HER2 alteration

\begin{tabular}{|c|c|c|c|c|}
\hline Characteristic & $\begin{array}{l}\text { HER2 altered } \\
\quad(\mathrm{n}=36)\end{array}$ & $\begin{array}{l}\text { Exon20 insertion } \\
\quad(n=22)\end{array}$ & $\begin{array}{c}H E R 2 \text { amplification } \\
(\mathrm{n}=15)\end{array}$ & p-value \\
\hline Age at diagnosis (yr) & 58 & 60.2 & 56.4 & \\
\hline Sex & & & & 0.093 \\
\hline Men & $20(55.6)$ & $10(45.5)$ & $11(73.3)$ & \\
\hline Women & $16(44.4)$ & $12(54.5)$ & $4(26.7)$ & \\
\hline ECOG PS & & & & 0.819 \\
\hline 0 & $4(11.1)$ & $3(13.6)$ & $1(6.7)$ & \\
\hline 1 & $30(83.3)$ & $18(81.8)$ & $13(86.7)$ & \\
\hline 2 & $2(5.6)$ & $1(4.5)$ & $1(6.7)$ & \\
\hline Tobacco & & & & 0.084 \\
\hline Never & $18(50.0)$ & $14(63.6)$ & $4(26.7)$ & \\
\hline Former & $11(30.6)$ & $5(22.7)$ & $6(40.0)$ & \\
\hline Current & $7(19.4)$ & $3(13.6)$ & $5(33.3)$ & \\
\hline Stage at diagnosis & & & & 0.753 \\
\hline I & $5(13.9)$ & $2(9.1)$ & $3(20.0)$ & \\
\hline II & $3(8.3)$ & $2(9.1)$ & $1(6.7)$ & \\
\hline III & $4(11.1)$ & $2(9.1)$ & $2(13.3)$ & \\
\hline IV & $24(66.7)$ & $16(72.7)$ & $9(60.0)$ & \\
\hline \multicolumn{5}{|l|}{ Metastasis sites } \\
\hline Bone & $21(58.3)$ & $14(63.6)$ & $8(53.3)$ & 0.531 \\
\hline Pleura & $18(50.0)$ & $12(54.5)$ & $6(40.0)$ & 0.385 \\
\hline Brain & $13(36.1)$ & $8(36.4)$ & $5(33.3)$ & 0.850 \\
\hline Contralateral metastasis & $11(30.6)$ & $6(27.3)$ & $5(33.3)$ & 0.728 \\
\hline Liver & $7(19.4)$ & $7(31.8)$ & 0 & 0.028 \\
\hline Distant LN & $7(19.4)$ & $4(18.2)$ & $3(20.0)$ & 0.808 \\
\hline Adrenal & $4(11.1)$ & $4(18.2)$ & 0 & 0.131 \\
\hline LMS & $3(8.3)$ & $2(9.1)$ & $1(6.7)$ & 1.000 \\
\hline Thyroid & $1(2.8)$ & $1(4.5)$ & 0 & 1.000 \\
\hline Histology & & & & 0.021 \\
\hline $\mathrm{ADC}$ & $32(88.9)$ & $22(100)$ & $11(73.3)$ & \\
\hline SQC & $4(11.1)$ & 0 & $4(26.7)$ & \\
\hline $\operatorname{EGFR(+)}$ & & & & 0.002 \\
\hline Wild type & $30(83.3)$ & $22(100)$ & $9(60.0)$ & \\
\hline Mutation & $6(16.7)$ & 0 & $6(40.0)$ & \\
\hline$A L K(+)$ & & & & 0.505 \\
\hline Wild type & $34(94.4)$ & $20(90.9)$ & $15(100)$ & \\
\hline Mutation & $2(5.6)$ & $2(9.1)$ & 0 & \\
\hline
\end{tabular}

Values are presented as number (\%). HER2, human epidermal growth factor receptor 2; ECOG PS, Eastern Cooperative Oncology Group performance status; LN, lymph node; LMS, leptomeningeal seeding; ADC, adenocarcinoma; SQC, squamous cell carcinoma; EGFR, epidermal growth factor receptor; ALK, anaplastic lymphoma kinase.

\section{Results}

A total of 1,108 patients diagnosed with NSCLC were screened in this study. Tissue samples from all patients were analyzed by NGS. The NGS results showed that 36 patients (3\%) had HER2 alteration. The median age of HER2-altered patients was 58 years (range, 35 to 79 years), $55.6 \%$ of the patients were male, and $50.0 \%$ were never-smokers. Adenocarcinoma was the predominant cancer type $(88.9 \%)$, and there were four cases (11.1\%) of squamous cell carcinoma. The most common metastatic site was bone (58.3\%), followed by pleura (50\%) and brain (36.1\%). At initial diagnosis, 66.7\% of patients were stage IV. Six patients $(16.7 \%)$ had a coexis- 
Table 2. Amino acid changes of HER2 in-frame insertion mutation group

\begin{tabular}{|c|c|c|c|}
\hline Patient No. & Amino acid change & Amino acid change & Total $(n=22)$ \\
\hline 1 & E770_A771insAYVM & E770_A771insAYVM & 12 \\
\hline 2 & E770_A771insAYVM & E770delinsEAYVM & 4 \\
\hline 3 & E770_A771insAYVM & G776delinsVC & 3 \\
\hline 4 & E770_A771insAYVM & V777delinsVGSP & 2 \\
\hline 5 & E770_A771insAYVM & A771_Y772insYVMA & 1 \\
\hline 6 & E770_A771insAYVM & - & - \\
\hline 7 & G776delinsVC & - & - \\
\hline 8 & E770_A771insAYVM & - & - \\
\hline 9 & E770_A771insAYVM & - & - \\
\hline 10 & A771_Y772insYVMA & - & - \\
\hline 11 & G776delinsVC & - & - \\
\hline 12 & E770_A771insAYVM & - & - \\
\hline 13 & E770_A771insAYVM & - & - \\
\hline 14 & E770_A771insAYVM & - & - \\
\hline 15 & E770delinsEAYVM & - & - \\
\hline 16 & G776delinsVC & - & - \\
\hline 17 & V777delinsVGSP & - & - \\
\hline 18 & E770delinsEAYVM & - & - \\
\hline 19 & E770delinsEAYVM & - & - \\
\hline 20 & V777delinsVGSP & - & - \\
\hline 21 & E770delinsEAYVM & - & - \\
\hline 36 & E770_A771insAYVM & - & - \\
\hline
\end{tabular}

HER2, human epidermal growth factor receptor 2.

Table 3. Best response to the first-line treatment of HER2 altered non-small cell lung cancer

\begin{tabular}{|c|c|c|}
\hline Treatment & No. $(\%)(n=36)$ & Best response \\
\hline Targeted therapy & $5(13.9)$ & \\
\hline Erlotinib & 1 & PR \\
\hline Gefitinib & 2 & $\mathrm{SD}(\mathrm{n}=2)$ \\
\hline Afatinib & 1 & PR \\
\hline Crizotinib & 1 & PD \\
\hline Cytotoxic chemotherapy & $28(77.8)$ & \\
\hline Pemetrexed+cisplatin & 23 & $\begin{array}{l}P R(n=6), S D(n=15) \\
P D(n=1), N / A(n=1)\end{array}$ \\
\hline Gemcitabine+cisplatin & 4 & PR $(n=3), S D(n=1)$ \\
\hline Pemetrexed monotherapy & 1 & $\mathrm{~N} / \mathrm{A}$ \\
\hline Immunotherapy & $3(8.3)$ & \\
\hline Durvalumab+tremelimumab+pemetrexed+cisplatin & 1 & SD \\
\hline Ipilimumab+nivolumab & 1 & SD \\
\hline Nivolumab & 1 & PR \\
\hline
\end{tabular}

HER2, human epidermal growth factor receptor 2; PR, partial response; SD, stable disease; PD, progressive disease; N/A, not acquired. 


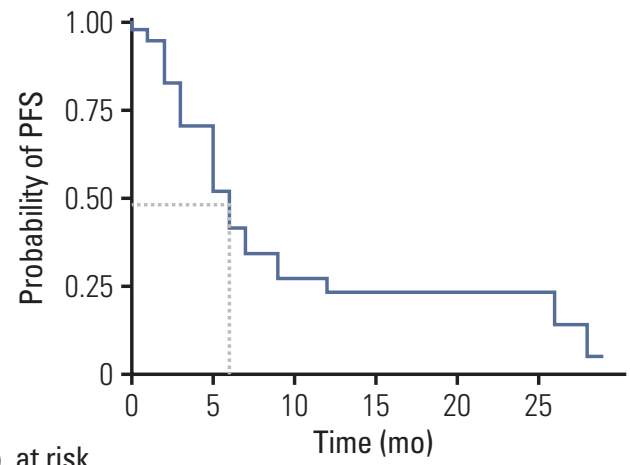

No. at risk

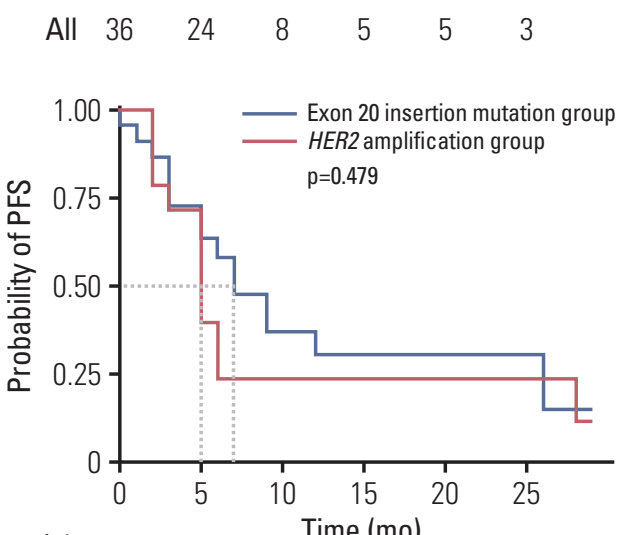

No. at risk

$\begin{array}{rllllll}\begin{array}{r}\text { Exon } 20 \text { insertion } \\ \text { mutation group }\end{array} & 22 & 16 & 6 & 3 & 3 & 2 \\ \begin{array}{r}\text { HER2 amplification } \\ \text { group }\end{array} & 15 & 9 & 3 & 3 & 3 & 2\end{array}$

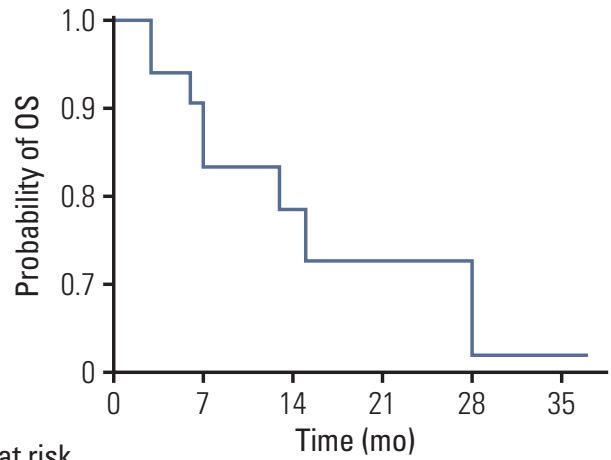

No. at risk

$\begin{array}{lllllll}\text { All } & 36 & 26 & 16 & 10 & 7 & 3\end{array}$

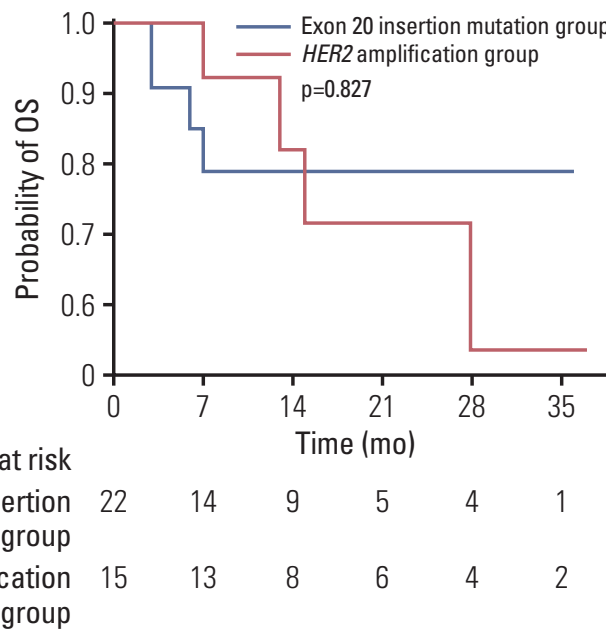

Fig. 1. Progression-free survival (PFS) and overall survival (OS) of human epidermal growth factor receptor 2 (HER2)-altered non-small cell lung cancer patients.

tent sensitizing EGFR mutation, and two patients (5.6\%) had ALK rearrangement (Table 1 ). The EGFR mutations consisted of three exon 21 L858R mutations, one exon 19 deletion, and two compound mutations (exon $18 \mathrm{E} 709 \mathrm{~K}+\mathrm{G} 719 \mathrm{~A}$ [n=1] and exon $18 \mathrm{G} 179 \mathrm{C}+$ exon 20 S768I [ $\mathrm{n}=1])$. Five of the six EGFR mutations $(83.3 \%)$ were detected by NGS. The other EGFR and two $A L K$ rearrangements were found by peptide nucleic acid-mediated polymerase chain reaction (PCR) clamping method and fluorescent in situ hybridization (FISH), respectively.

Among the 36 HER2-altered NSCLC patients, 22 patients $(61.1 \%)$ had exon 20 in-frame insertion mutation, 15 patients (41.7\%) had HER2 gene amplification, and one patient had both. The most frequently inserted amino acid sequence was E770_A771insAYVM (55\%), followed by E770delinsEAYVM, G776delinsVC, and V777delinsVGSP (Table 2). Demographics, including Eastern Cooperative Oncology Group performance status, stage at diagnosis, and the $A L K$ rearrangement incidence were similar between the exon 20 in-frame inser- tion mutation group and the HER2 amplification group. However, patients with adenocarcinoma were significantly more predominant in the exon 20 in-frame insertion mutation group than the HER2 amplification group (100\% vs. $73.3 \%$, respectively, $\mathrm{p}=0.021$ ). The proportion of never-smokers and frequency of metastasis to the liver were also higher in the exon 20 in-frame insertion mutation group $(63.6 \%$ vs. $26.7 \%, \mathrm{p}=0.027 ; 31.8 \%$ vs. $0 \%, \mathrm{p}=0.025$, respectively). In contrast, EGFR mutation was more frequent in the HER2 amplification group ( $40 \%$ vs. $0 \%, p=0.002)$, and the proportion of male patients tended to be higher in the HER2 amplification group $(73.3 \%$ vs. $45.5 \%, \mathrm{p}=0.093)$, although the difference was not statistically significant (Table 1 ).

As the initial treatment, 28 patients $(77.8 \%)$ were treated with cytotoxic chemotherapy (pemetrexed/ cisplatin [ $\mathrm{n}=23]$, gemcitabine/cisplatin $[n=4]$, pemetrexed monotherapy $[\mathrm{n}=1])$, and the median PFS of these patients was six months (95\% confidence interval [CI], 3.969 to 8.031 ). Of the six patients with coexisting sensitizing EGFR mutations, four 


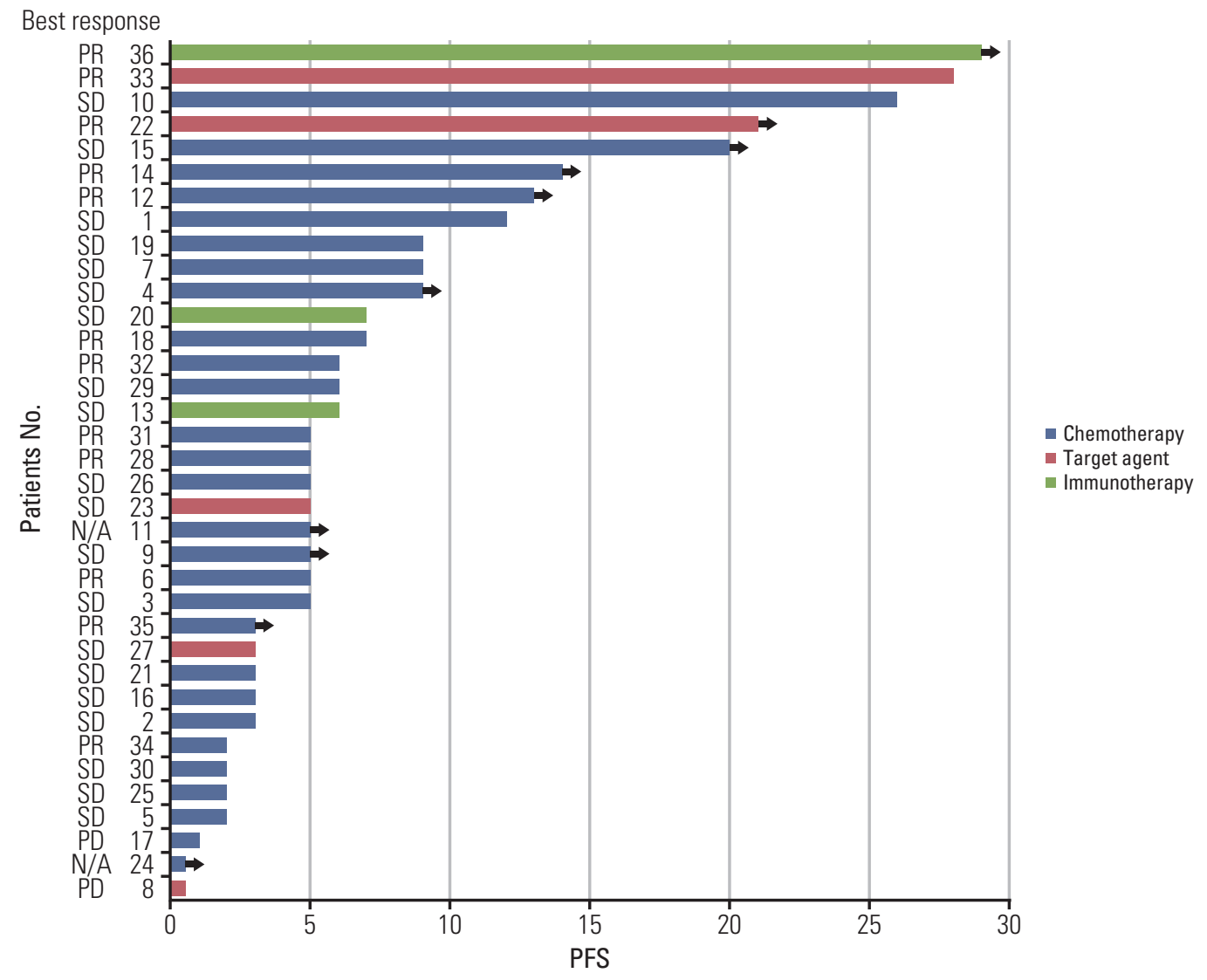

Fig. 2. Swimmers plot of progression-free survival (PFS) of first-line treatment. PR, partial response; SD, stable disease; $\mathrm{N} / \mathrm{A}$, not acquired.

patients were treated with EGFR tyrosine kinase inhibitors (TKIs) as first-line treatment (erlotinib [n=1], gefitinib [n=2], or afatinib [n=1]). Two patients showed PFS of 5 months and 3 months and achieved the best response of stable disease. Another two patients showed PFS of more than 21 months with partial response. The other two patients with EGFR mutations were treated with EGFR-TKI (gefitinib [n=2]) as second-line treatment, and the PFS were 33 and 15 months, respectively. One of the two patients with $A L K$ rearrangement was initially treated with crizotinib, and the PFS was one month.

Of the 28 patients without coexisting sensitizing EGFR mutation or ALK rearrangement, three (two with exon 20 insertion mutation, one with both HER2 alterations) were able to participate in a clinical trial and were treated with immunotherapy (durvalumab / tremelimumab / pemetrexed / cisplatin [n=1], ipilimumab/nivolumab [n=1], nivolumab $[\mathrm{n}=1]$ ) (Table 3). Two of these patients showed PFS of 6 months and 7 months, and one patient showed sustained treatment response for 29 months until the data cut-off date. Overall, with 14 months of median follow-up (95\% CI, 8.039 to 19.961), the median PFS of first-line treatment was six months (95\% CI, 4.172 to 7.828), and the median OS had not been reached. There was no statistically significant difference between the exon 20 in-frame insertion mutation group and HER2 amplification group in terms of PFS or OS (PFS, 7 months vs. 5 months, respectively; $\mathrm{p}=0.479$ ) (Fig. 1).

\section{Discussion}

In our study, HER2-altered NSCLC was associated with a higher rate of adenocarcinoma (88.9\%) and never-smokers $(50 \%)$. This result is consistent with previous studies performed in Europe and Asia [3-5]. By contrast, there was no relationship between HER2 alteration and sex, unlike the previous studies. Given that previous studies included smaller numbers of patients and were based on results from FISH or PCR, we could emphasize closer associations of adenocarcinoma histology and smoking history with the incidence of 
HER2-altered NSCLC based on our NGS results. Early studies described HER2 mutations as mutually exclusive with other oncogenic driver mutations, such as EGFR mutations [6]. However, recent reports suggested that concomitant EGFR mutation or ALK rearrangement is more common [9], and we found a relatively high rate of coexistence in this study (22.2\%). Most of coexistent EGFR mutations (83.3\%) were detected by NGS solely, not PCR. NGS seems to have better sensitivity in detecting EGFR mutations, which can be a key target for treatment. On the other hand, one EGFR mutation and two ALK rearrangements were found in PCR and FISH, respectively, not NGS. It means NGS is not a perfect test, and we need a better NGS panel to detect broader mutations, including rare ones.

HER2 alteration consists of HER2 amplification and HER2 mutation, which have been revealed as in-frame insertion mutation. Li et al. [10] showed that HER2 mutations were not associated with HER2 amplification and suggested a distinct disease entity and therapeutic target for each alteration. Of the 36 HER2-altered patients in our study, 35 patients had either HER2 mutation or HER2 amplification. One patient harbored both gene alterations. We found distinct clinical features between the HER2 mutation and HER2 amplification groups. The proportions of never-smokers, adenocarcinoma, and metastasis to the liver were more frequent in the exon 20 in-frame insertion mutation group, while the HER2 amplification group showed a high proportion of EGFR mutation. Although these discrepancies should be confirmed with a bigger study, their different clinical features imply different pathogenesis and treatment strategies in NSCLC.

Most of the HER2-altered patients $(77.8 \%)$ were treated with cytotoxic chemotherapy as first-line treatment due to the unavailability of targeted agents. The median PFS of the patients was 6 months, which was similar to previous studies [11]. Other patients who were treated with EGFR-TKI, ALK inhibitor, or immunotherapy showed diverse responses (Fig. 2). There was no significant difference between the HER2 mutation group and the HER2 amplification group in terms of clinical outcomes.

Many clinical trials have attempted to find the best treatment for HER2-altered NSCLC. Although most of the studies used HER2 TKI or HER2-targeted monoclonal antibody (trastuzumab), which yielded disappointing results, some recent studies have produced encouraging results. A phaseII basket trial showed a therapeutic benefit of adotrastuzumab emtansine (T-DM1) for NSCLC patients with HER2 mutations, who achieved a median 5-month PFS with acceptable toxicity [12]. Although the study size was small, based on this result, National Comprehensive Cancer Network guideline suggests T-DM1 as an available targeted agent for HER2-mutant NSCLC. In addition, afatinib and dacomitinib, which are pan-HER inhibitors and have been approved as first-line treatments for metastatic EGFR-mutant NSCLC, have shown promising results in phase-II studies $[13,14]$. Ogoshi [15] also showed that neratinib inhibited the growth of HER2-altered NSCLC cell lines. Other therapies for HER2 alteration in lung cancer are still being introduced [16,17], and in clinical trials (NCT03505710, NCT02535507).

In our study, two patients showed an outstanding therapeutic response. One patient (No. 22 in Fig. 2) harboring HER2 amplification with L858R mutation was treated with afatinib and showed a partial response after initial therapy. The therapeutic effect was sustained until the data cut-off date, for 21 months. Even considering the EGFR mutation, the PFS was much longer than the results of previous LUXlung trials $[18,19]$. Previously, Fang et al. [20] showed a divergent therapeutic benefit from afatinib according to mutation type of HER2-altered NSCLC. Patients with G776delinsVC and G778_P780dup derive the greatest benefit from afatinib, while patients with A775_G776insYVMA, co-mutations in the TP53 and PI3K/AKT/mammalian target of rapamycin (mTOR) pathway show primary resistance to afatinib [20]. One preclinical study reported the efficacy of afatinib for NSCLC with HER2 amplification [21]. Although further research is needed, based on our experience, we suggest that afatinib seems to have a sustained therapeutic effect for NSCLC patients with HER2 amplification.

Another patient (No. 36 in Fig. 2) was treated with nivolumab and showed the most significant PFS of more than 29 months. The patient did not have any EGFR mutations, ALK rearrangement, or programmed death 1 / programmed deathligand 1 overexpression. Interestingly, he had both HER2 amplification and exon 20 insertion mutation, and the inserted amino acid was E770_A771insAYVM. One previous case report showed that a HER2 mutant patient with exon 20 insertion (M774_A775insAYVM) exhibited a great response to nivolumab [22]. However, the coexistence of HER2 mutation and amplification has been reported with low incidence in several studies $[3,23,24]$, and HER2 amplification was assessed by different methods in each study, which could have a big impact on results and interpretation [10]. Therefore, the exact roles of the coexistence and certain mutation types of $H E R 2$ require further study.

This study has one important limitation. Given the high incidence of EGFR mutations in Korea, a reflex test for EGFR and $A L K$ alterations is routine practice, while NGS is performed mostly for EGFR / ALK-negative patients. Therefore, HER2-alteration incidence might have been overestimated in this study. Nevertheless, this is the biggest study to evaluate the clinical features of HER2-altered NSCLC in an Asian population, as detected by NGS.

In conclusion, NSCLC patients with HER2 alterations have distinct clinical features, and NSCLC patients with HER2 in-frame insertion mutation and HER2 amplification exhib- 
ited unique characteristics. Given the limited number of available effective agents to target HER2-altered NSCLC, further investigation with novel agents is needed.

\section{Electronic Supplementary Material}

Supplementary materials are available at Cancer Research and Treatment website (https:// www.e-crt.org).

\section{Conflicts of Interest}

Conflict of interest relevant to this article was not reported.

\section{References}

1. Shigematsu H, Takahashi T, Nomura M, Majmudar K, Suzuki $\mathrm{M}$, Lee $\mathrm{H}$, et al. Somatic mutations of the HER2 kinase domain in lung adenocarcinomas. Cancer Res. 2005;65:1642-6.

2. Yu HA, Planchard D, Lovly CM. Sequencing therapy for genetically defined subgroups of non-small cell lung cancer. Am Soc Clin Oncol Educ Book. 2018;38:726-39.

3. Mazieres J, Peters S, Lepage B, Cortot AB, Barlesi F, BeauFaller $\mathrm{M}$, et al. Lung cancer that harbors an HER2 mutation: epidemiologic characteristics and therapeutic perspectives. J Clin Oncol. 2013;31:1997-2003.

4. Li C, Sun Y, Fang R, Han X, Luo X, Wang R, et al. Lung adenocarcinomas with HER2-activating mutations are associated with distinct clinical features and HER2/EGFR copy number gains. J Thorac Oncol. 2012;7:85-9.

5. Arcila ME, Chaft JE, Nafa K, Roy-Chowdhuri S, Lau C, Zaidinski $\mathrm{M}$, et al. Prevalence, clinicopathologic associations, and molecular spectrum of ERBB2 (HER2) tyrosine kinase mutations in lung adenocarcinomas. Clin Cancer Res. 2012;18: 4910-8.

6. Pillai RN, Behera M, Berry LD, Rossi MR, Kris MG, Johnson $\mathrm{BE}$, et al. HER2 mutations in lung adenocarcinomas: a report from the Lung Cancer Mutation Consortium. Cancer. 2017; 123:4099-105.

7. Shin HT, Choi YL, Yun JW, Kim NKD, Kim SY, Jeon HJ, et al. Prevalence and detection of low-allele-fraction variants in clinical cancer samples. Nat Commun. 2017;8:1377.

8. Williams HL, Walsh K, Diamond A, Oniscu A, Deans ZC. Validation of the Oncomine $\left({ }^{\mathrm{TM}}\right)$ focus panel for next-generation sequencing of clinical tumour samples. Virchows Arch. 2018; 473:489-503.

9. Mazieres J, Barlesi F, Filleron T, Besse B, Monnet I, Beau-Faller $\mathrm{M}$, et al. Lung cancer patients with HER2 mutations treated with chemotherapy and HER2-targeted drugs: results from the European EUHER2 cohort. Ann Oncol. 2016;27:281-6.

10. Li BT, Ross DS, Aisner DL, Chaft JE, Hsu M, Kako SL, et al. HER2 amplification and HER2 mutation are distinct molecular targets in lung cancers. J Thorac Oncol. 2016;11:414-9.

11. Scagliotti GV, Parikh P, von Pawel J, Biesma B, Vansteenkiste J, Manegold C, et al. Phase III study comparing cisplatin plus gemcitabine with cisplatin plus pemetrexed in chemotherapynaive patients with advanced-stage non-small-cell lung can- cer. J Clin Oncol. 2008;26:3543-51.

12. Li BT, Shen R, Buonocore D, Olah ZT, Ni A, Ginsberg MS, et al. Ado-trastuzumab emtansine for patients with HER2mutant lung cancers: results from a phase II basket trial. J Clin Oncol. 2018;36:2532-7.

13. Lai WC, Lebas L, Milia J, Barnes TA, Gautschi O, Peters S, et al. Afatinib in patients with metastatic HER2-mutant lung cancers: an international multicenter study. J Clin Oncol. 2017; 35(15 Suppl):9071.

14. Garrido-Castro AC, Felip E. HER2 driven non-small cell lung cancer (NSCLC): potential therapeutic approaches. Transl Lung Cancer Res. 2013;2:122-7.

15. Ogoshi Y. P3.13-35 Antitumor effect of neratinib targeting HER2-altered lung cancer. J Thorac Oncol. 2018;13(10 Suppl): S990.

16. Chouitar J, Vincent S, Brake R, Li S. P2.13-32 TAK-788 is a novel and potent tyrosine kinase inhibitor with selective activity against EGFR/HER2. J Thorac Oncol. 2018;13(10 Suppl): S811.

17. Liu S, Li S, Hai J, Wang X, Chen T, Quinn MM, et al. Targeting HER2 aberrations in non-small cell lung cancer with osimertinib. Clin Cancer Res. 2018;24:2594-604.

18. Sequist LV, Yang JC, Yamamoto N, O'Byrne K, Hirsh V, Mok $\mathrm{T}$, et al. Phase III study of afatinib or cisplatin plus pemetrexed in patients with metastatic lung adenocarcinoma with EGFR mutations. J Clin Oncol. 2013;31:3327-34.

19. Wu YL, Zhou C, Hu CP, Feng JF, Lu S, Lu YH, et al. LUX-Lung 6: a randomized, open-label, phase III study of afatinib (A) versus gemcitabine/cisplatin (GC) as first-line treatment for Asian patients (pts) with EGFR mutation-positive (EGFR M+) advanced adenocarcinoma of the lung. J Clin Oncol. 2013; 31(15 Suppl):8016.

20. Fang W, Zhao S, Liang Y, Yang Y, Yang L, Dong X, et al. P088 Distribution of different HER2 mutations and their impact on clinical responses to afatinib in HER2-mutant lung cancer. J Thorac Oncol. 2018;13(12 Suppl):S1080.

21. Suzawa K, Toyooka S, Sakaguchi M, Morita M, Yamamoto H, Tomida S, et al. Antitumor effect of afatinib, as a human epidermal growth factor receptor 2-targeted therapy, in lung cancers harboring HER2 oncogene alterations. Cancer Sci. 2016; 107:45-52. 
22. Catania C, Passaro A, Rocco EG, Spitaleri G, Barberis M, Noberasco $C$, et al. Dramatic antitumor activity of nivolumab in advanced HER2-positive lung cancer. Clin Lung Cancer. 2016;17:e179-83.

23. Yoshizawa A, Sumiyoshi S, Sonobe M, Kobayashi M, Uehara T, Fujimoto M, et al. HER2 status in lung adenocarcinoma: a comparison of immunohistochemistry, fluorescence in situ hybridization (FISH), dual-ISH, and gene mutations. Lung Cancer. 2014;85:373-8.

24. Suzuki M, Shiraishi K, Yoshida A, Shimada Y, Suzuki K, Asamura $\mathrm{H}$, et al. HER2 gene mutations in non-small cell lung carcinomas: concurrence with Her2 gene amplification and Her2 protein expression and phosphorylation. Lung Cancer. $2015 ; 87: 14-22$ 